\title{
Changes of serum miR-223-3p in patients with oral cancer treated with TPF regimen and the prognosis
}

\author{
$\mathrm{CHUNRU} \mathrm{LI}^{1 *}$, YAO FENG ${ }^{1 *}$ and WEIRAN SHAO ${ }^{2}$ \\ Departments of ${ }^{1}$ Endodontics and ${ }^{2}$ Emergency, Affiliated Stomatological Hospital of Jiamusi University, \\ Jiamusi, Heilongjiang 154002, P.R. China
}

Received September 18, 2019; Accepted November 14, 2019

DOI: $10.3892 / \mathrm{ol} .2020 .11258$

\begin{abstract}
Changes of serum miR-223-3p in patients with oral cancer treated with TPF regimen and the prognosis were investigated. Fifty patients with oral cancer treated in the Affiliated Stomatological Hospital of Jiamusi University from March 2014 to January 2016 were enrolled in the study group, while 50 healthy subjects receiving physical examinations during the same period were enrolled in the control group. Serum expression of miR-223-3p was quantified by RT-qPCR. The diagnostic value of miR-223-3p in oral cancer was analyzed by the receiver operating characteristic (ROC) curve. Expression of miR-223-3p before and after treatment was compared. The study group was divided into the remission and the non-remission group based on the treatment outcome to analyze the predictive value of miR-223-3p. Patients were followed up for 3 years. Cox regression analysis was performed to analyze the independent prognostic factors. The relative serum miR-223-3p level was lower in the study than in the control group $(\mathrm{P}<0.001)$. Expression of miR-223-3p was significantly higher after treatment than before $(\mathrm{P}<0.05)$. Spearman's correlation analysis indicated that miR-223-3p expression before treatment gradually increased with the improvement of treatment outcome $(r=0.617, \mathrm{P}<0.001)$. The miR-223-3p level was markedly higher in the remission than in the non-remission group $(\mathrm{P}<0.05)$. The area under the ROC curve of miR-223-3p was 0.797. Multivariate Cox regression analysis demonstrated that the degree of differentiation [HR: 11.862 (95\% CI: 2.730-51.547)] and miR-223-3p [HR: 3.489 (95\% CI: 1.447-8.413)] were independent prognostic factors. The 3-year survival of patients with high differentiation and high miR-223-3p expression was significantly higher than that of patients with poor differentiation and low miR-223-3p
\end{abstract}

Correspondence to: Dr Weiran Shao, Department of Emergency, Affiliated Stomatological Hospital of Jiamusi University, 522 Hongqi Road, Jiamusi, Heilongjiang 154002, P.R. China

E-mail: r3p9cg@163.com; shaoweiran1976@163.com

${ }^{*}$ Contributed equally

Key words: TPF regimen, oral cancer, miR-223-3p expression $(\mathrm{P}<0.05)$. In conclusion, $\mathrm{miR}-223-3 \mathrm{p}$ expression is low in oral cancer, and it shows potential for predicting the efficacy and prognosis of patients with oral squamous cell carcinoma (OSCC) after TPF regimen.

\section{Introduction}

Oral cancer is a common oral and maxillofacial malignant tumor, among which oral squamous cell carcinoma (OSCC) is the most prevalent $(1,2)$. The reported annual new cases of OSCC are over 300,000 (3). The diagnosis and treatment of OSCC are advancing due to the development in medical fields, but the prognosis is not very good. Data show that the 5-year survival rate of OSCC is only 50 to $60 \%$, and some patients have advanced disease and local metastasis at the time of diagnosis, which is not conducive to clinical treatment $(4,5)$. A previous study stated that the patients with advanced oral cancer had a poor 3-year survival rate (6). At present, the most effective treatment for patients with locally advanced OSCC suitable for resection is comprehensive treatment based on radical surgery $(7)$.

In recent years, chemotherapy induced by TPF regimen (Docetaxel + Cisplatin + Fluorouracil) has been a hotpot of clinical research of oral cancer (8). TPF regimen helps to downsize the tumor and reduce the difficulty of radical operation (9). The present clinical evaluation of solid tumors mainly depends on imaging tests, which, unlike serological tests, have a high economic cost and high difficulty in sampling, as well as certain impacts on the patient's body. Therefore, the search for a serological index to evaluate the efficacy and prognosis of patients after treatment is crucial (10). The microRNA (miR), a non-coding short-stranded RNA, 21 to 25 nt long, can bind to the target gene 3'UTR by complementary pairing to degrade or inhibit the expression of the target gene (11). Several studies have found that $\mathrm{miR}$ is differentially expressed in lung, liver, breast, oral cancer, and other cancers, and is expected to be an indicator of cancer observation and prognosis (12-15). As a member of the miR family, miR-223 was reported to be differentially expressed in oral cancer in the study by Tachibana et al (16), and it has potential to become a diagnostic and therapeutic target in oral cancer. The study by Soga et al (17) analyzed the miR expression profile of oral squamous cell carcinoma and discovered a low miR-223 expression in patients with oral squamous cell carcinoma. 
However, the potentiality of miR-223 to function as a shortterm efficacy predictor and long-term prognostic index after chemotherapy has not been studied.

This study observed the expression of miR-223-3p in patients treated with TPF chemotherapy and explored its value in predicting the efficacy of OSCC patients, aiming to provide a clinical reference.

\section{Patients and methods}

Sample collection. Fifty patients with oral cancer treated in the Affiliated Stomatological Hospital of Jiamusi University (Jiamusi, China) from March 2014 to January 2016 were enrolled in the study group (aged 50-73 years), while 50 healthy subjects receiving physical examinations during the same period in the hospital were enrolled in the control group. This study was approved by the Medical Ethics Committee of the hospital.

Inclusion criteria: Patients aged $>18$ years and diagnosed with OSCC by imaging and pathological biopsy; patients at stage III and IV according to TNM staging system; patients in line with the 8th edition of the American Joint Committee on Cancer (AJCC) Cancer Staging Manual issued in 2017 (18); patients willing to cooperate with the treatment and follow-up.

Exclusion criteria: Patients with other tumors or congenital defects in liver, kidney, and heart functions; patients with estimated survival time of less than 1 month; patients with infections before the admission, patients intolerant of drugs of this treatment; patients receiving no relevant targeted anticancer treatments before this treatment.

Main instruments and drugs. Docetaxel was from Shanghai Acebright Pharmaceuticals Co., Ltd., China. Cisplatin was from Guizhou Hanfang Pharmaceutical Co., Ltd., China. Fluorouracil was from Hainan Choitec Pharmaceuticals Co., Ltd., China. TRIzol reagent and the mirVana ${ }^{\mathrm{TM}} \mathrm{RT}$-qPCR miRNA detection kit were purchased from Invitrogen, Carlsbad, CA, USA (15590618, AM1558). TaqMan ${ }^{\mathrm{TM}}$ microRNA reverse transcription kit and the PCR instrument were purchased from Applied Biosystems, Foster, CA, USA (4366596, 4427975, 7500).

Treatment methods. Patients were treated with TPF regimen and oral radical surgery as follows: $75 \mathrm{mg} / \mathrm{m}^{2}$ of docetaxel (d1), $75 \mathrm{mg} / \mathrm{m}^{2}$ of cisplatin (d1), $750 \mathrm{mg} / \mathrm{m}^{2}$ of 5 -Fluorouracil (d1-d5). One treatment cycle comprised of 19 days and one course comprised of two cycles. The efficacy was evaluated 1 week after the chemotherapy. Radical resection of oral cancer was performed 2 weeks after the chemotherapy.

Detection of miR-223-3p expression. A total of $5 \mathrm{ml}$ of peripheral venous blood was collected from all subjects before and after the treatment. Thirty minutes later, the blood was centrifuged at $1,500 \mathrm{x}$ g at $24^{\circ} \mathrm{C}$ for $10 \mathrm{~min}$ to obtain the serum for total RNA extraction with TRIzol reagent. The purity, concentration, and integrity of total RNA were measured by UV spectrophotometer and agarose gel electrophoresis. Total RNA was reverse transcribed using the TaqMan ${ }^{\mathrm{TM}}$ microRNA reverse transcription kit in line with the kit instructions. The miR-223-3p expression in the collected cDNA was detected by mirVana $^{\mathrm{TM}} \mathrm{RT}$-qPCR miRNA detection kit and the 7500 PCR instrument. The detection system consisted of $5 \mu \mathrm{l}$ of mirVana $5 \mathrm{X}$ PCR Buffer, $0.5 \mu \mathrm{l}$ of $50 \mathrm{X} \mathrm{ROX}^{\mathrm{TM}}, 1 \mu \mathrm{l}$ of cDNA, $0.5 \mu \mathrm{l}$ of forward primer, $0.5 \mu \mathrm{l}$ of reverse primer, and enough nuclease-free water to add the system up to $20 \mu$ l. Amplification conditions: 40 cycles of pre-denaturation at $95^{\circ} \mathrm{C}$ for $3 \mathrm{~min}$, denaturation at $95^{\circ} \mathrm{C}$ for $15 \mathrm{sec}$, annealing and extension at $60^{\circ} \mathrm{C}$ for $30 \mathrm{sec}$. Three replicate wells were set for each sample, and the experiment was carried out 3 times. In the present study, U6 was used as an internal reference, and the data were analyzed using $2^{-\Delta \Delta \mathrm{Cq}}(19)$.

Follow-up. The survival of patients was followed up for 3 years by telephone and clinical re-examination, and the follow-up was performed every 3 months.

\section{Outcome measures}

Main outcome measures. The expression of miR-223-3p in the study and the control group and the expression of miR-223-3p before and after treatment in the study group were monitored. The short-term clinical efficacy after treatment was divided into complete response (CR), partial response (PR), stable disease (SD), and progressive disease (PD) according to the response evaluation criteria in solid tumor by WHO (20). Patients with CR and PR were in remission, while patients with SD and PD were not in remission.

Secondary outcome measures. Patients were divided into the remission group and the non-remission group based on efficacy. The predictive value of miR-223-3p for the efficacy was compared between the two groups before the treatment. Kaplan-Meier survival curve was used to analyze the relationship between miR-223-3p high/low expression (median value: 3.22) and OSCC survival, and Cox regression analysis was performed to analyze OSCC prognosis.

Statistical analysis. The collected data were statistically analyzed using the SPSS20.0 software and visualized using the GraphPad 7 software. The distribution of the measurement data were analyzed using the Kolmogorov-Smirnov test. The measurement data with normal distribution were expressed as the mean \pm standard deviation (mean $\pm \mathrm{SD}$ ), the comparison between groups was performed using the independent sample $\mathrm{t}$-test and the comparison within the group was performed by paired t-test. The count data were analyzed by the chi-square test. Spearman's test was used to analyze the relationship between miR-223-3p and clinical efficacy. ROC curve was employed to observe the diagnostic value. Kaplan-Meier survival curve was used to display the 3-year survival which was analyzed by the log-rank test. Multivariate Cox regression was conducted to explore the independent risk factors affecting the prognosis of patients. A statistical difference was recognized at $\mathrm{P}<0.05$.

\section{Results}

Baseline data analysis. The study and the control group were not statistically different in sex, age, BMI, medical history, smoking, drinking, and place of residence $(\mathrm{P}>0.05)$ (Table I). 
Table I. Baseline data analysis.

\begin{tabular}{|c|c|c|c|c|}
\hline Factors & Study group $(n=50)$ & Control group $(n=50)$ & $\chi^{2} / \mathrm{t}$-test & P-value \\
\hline \multicolumn{5}{|l|}{ Sex } \\
\hline Male & $31(62.00)$ & $35(70.00)$ & 0.713 & 0.398 \\
\hline Female & $19(38.00)$ & $15(30.00)$ & & \\
\hline Age (year) & $62.4 \pm 5.1$ & $61.2 \pm 5.7$ & 1.109 & 0.270 \\
\hline BMI $\left(\mathrm{kg} / \mathrm{m}^{2}\right)$ & $22.51 \pm 2.25$ & $22.84 \pm 1.72$ & 0.824 & 0.412 \\
\hline \multicolumn{5}{|l|}{ Medical history } \\
\hline Hypertension & $18(36.00)$ & $14(28.00)$ & 0.735 & 0.391 \\
\hline Diabetes & $12(24.00)$ & $9(18.00)$ & 0.543 & 0.461 \\
\hline Hyperlipidemia & $8(46.00)$ & $5(10.00)$ & 0.796 & 0.372 \\
\hline \multicolumn{5}{|l|}{ Smoking } \\
\hline Yes & $30(60.00)$ & $34(68.00)$ & 0.694 & 0.405 \\
\hline No & $20(40.00)$ & $16(32.00)$ & & \\
\hline \multicolumn{5}{|l|}{ Drinking } \\
\hline Yes & $7(14.00)$ & $4(8.00)$ & 0.919 & 0.338 \\
\hline No & $43(86.00)$ & $46(92.00)$ & & \\
\hline \multicolumn{5}{|l|}{ Place of residence } \\
\hline Urban area & $35(70.00)$ & $40(80.00)$ & 1.333 & 0.248 \\
\hline Rural area & $15(30.00)$ & $10(20.00)$ & & \\
\hline \multicolumn{5}{|l|}{ Location of tumor } \\
\hline Glossa & $22(44.00)$ & & & \\
\hline Other & $28(56.00)$ & & & \\
\hline \multicolumn{5}{|l|}{ Clinical stage } \\
\hline III & $35(70.00)$ & & & \\
\hline IV & $15(30.00)$ & & & \\
\hline \multicolumn{5}{|l|}{ Degree of differentiation } \\
\hline High differentiation & $18(36.00)$ & & & \\
\hline Moderate differentiation & $32(64.00)$ & & & \\
\hline
\end{tabular}

Table II. Analysis of 3-year prognostic factors.

\begin{tabular}{|c|c|c|c|c|c|c|}
\hline \multirow[b]{2}{*}{ Parameter } & \multicolumn{3}{|c|}{ Univariate Cox } & \multicolumn{3}{|c|}{ Multivariate Cox } \\
\hline & P-value & HR & $95 \% \mathrm{CI}$ & P-value & HR & $95 \% \mathrm{CI}$ \\
\hline Sex (male vs. female) & 0.510 & 0.752 & $0.322 \sim 1.758$ & & & \\
\hline Age $(\leq 60$ vs. $>60)$ & 0.896 & 0.943 & $0.391 \sim 2.275$ & & & \\
\hline Location of tumor (glossa vs. other) & 0.750 & 1.141 & $0.506 \sim 2.573$ & & & \\
\hline Clinical stage (III vs. IVA) & 0.219 & 1.679 & $0.734 \sim 3.839$ & & & \\
\hline Degree of differentiation (high vs. moderate) & 0.049 & 2.699 & $1.055 \sim 7.246$ & 0.031 & 2.988 & $1.106 \sim 8.071$ \\
\hline miR-223-3p expression (high vs. low) & 0.024 & 2.677 & $1.14 \sim 6.287$ & 0.014 & 2.933 & $1.241 \sim 6.934$ \\
\hline
\end{tabular}

HR, hazard ratio; CI, confidence interval.

Expression and efficacy predictive value of $m i R-223-3 p$ in patients. According to the detection of serum miR-223-3p expression in the study and the control group, the relative serum miR-223-3p level was lower in the study than in the control group $(\mathrm{P}<0.001)$. The miR-223-3p expression in the study group after the treatment was significantly higher than before $(\mathrm{P}<0.05)$. After the treatment, no great difference was observed between the study group and the control group in the miR-223-3p expression ( $\mathrm{P}>0.05)$.

The treatment outcome was CR in 4 patients, $P R$ in 26, SD in 17, and PD in 3. Spearman's correlation analysis indicated that miR-223-3p expression gradually increased with the 
A

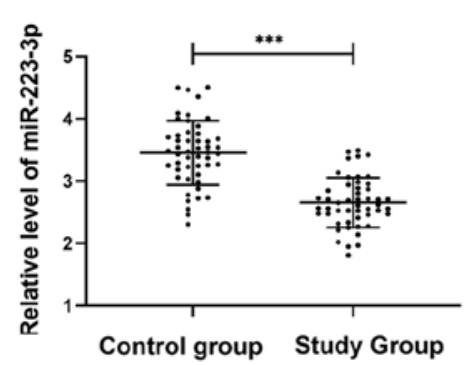

D

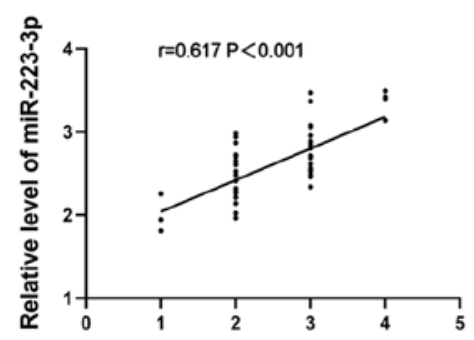

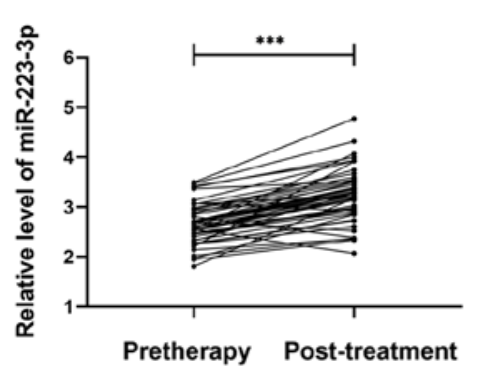

E

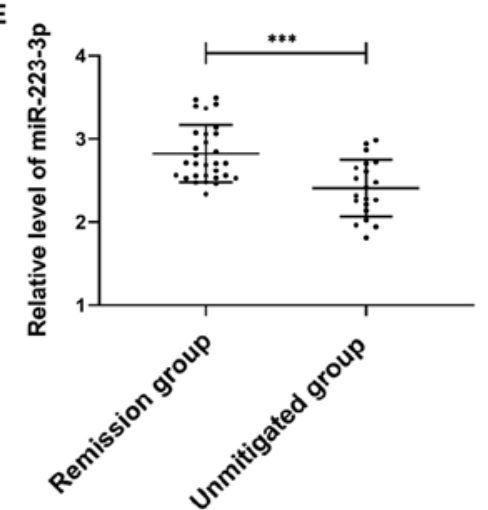

C

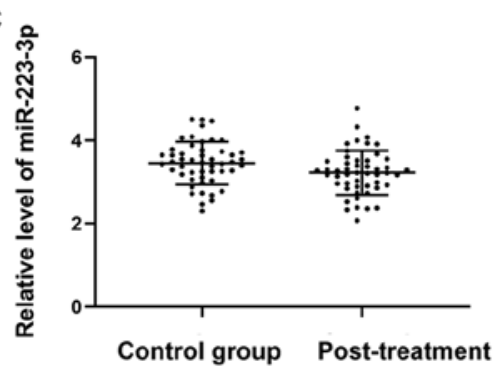

$\mathbf{F}$

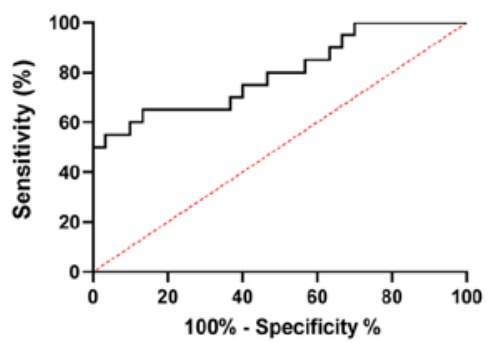

Figure 1. Expression and efficacy predictive value of miR-223 in OSCC. (A) Expression of miR-223-3p in OSCC patients. (B) miR-223-3p expression before and after the treatment in OSCC patients. (C) After the treatment, miR-223-3p expression in OSCC patients was not significantly different from that in healthy subjects in the control group. (D) Correlation between miR-223-3p and clinical efficacy. (E) miR-223-3p expression in the remission group and the non-remission group. (F) miR-223-3p prediction of the clinical efficacy of OSCC patients before the treatment. When the cut-off value was 2.527 , the best specificity was $86.67 \%$, sensitivity was $65.00 \%$, and Youden index was $51.67 \%$. OSCC, oral squamous cell carcinoma. ${ }^{* * *} \mathrm{P}<0.001$

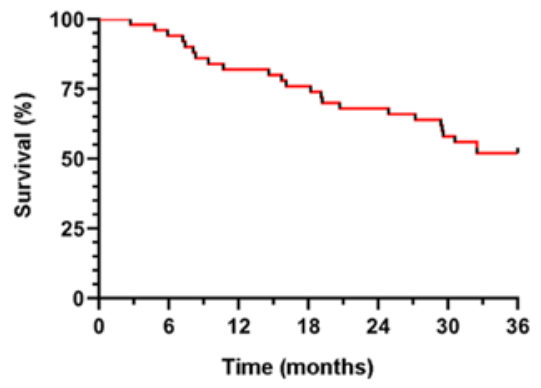

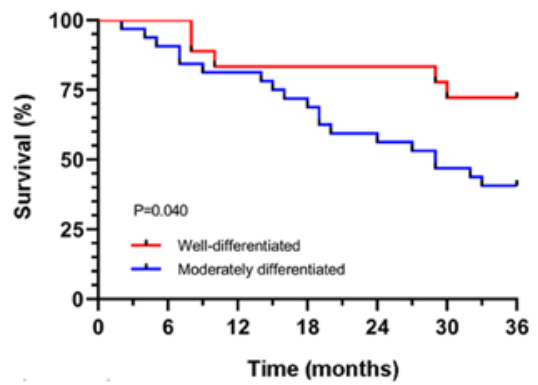

C

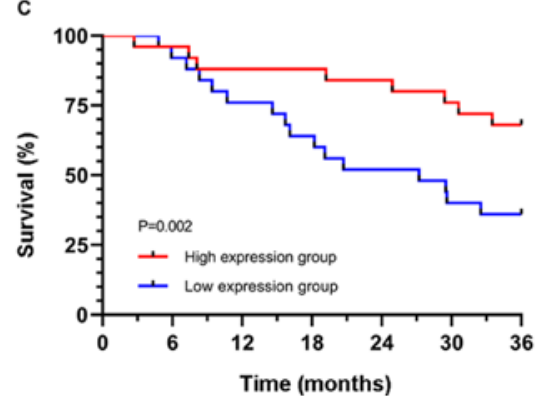

Figure 2. Survival curve of independent prognostic factors. (A) Three-year survival of patients. (B) Three-year survival of patients with different differentiation. (C) Three-year survival of patifents with different miR-223-3p expression.

improvement of treatment outcome $(\mathrm{r}=0.617, \mathrm{P}<0.001)$. Patients were divided into the remission group $(\mathrm{n}=30)$ and the non-remission group $(\mathrm{n}=20)$ according to the treatment outcome. The miR-223-3p level before the treatment was markedly higher in the remission group than in the non-remission group $(\mathrm{P}<0.05)$. The area under the ROC curve of miR-223-3p was 0.797 (21) (Fig. 1).

Relationship between miR-223-3p and the prognosis and survival of patients. All 50 patients were followed up for three 
years. The number of surviving patients was 26 , with a survival rate of $52.00 \%$. Univariate Cox regression analysis indicated that the degree of differentiation and miR-223-3p expression were prognostic factors. Multivariate Cox regression analysis demonstrated that the degree of differentiation [HR: 11.862 (95\% CI: 2.730-51.547)] and miR-223-3p [HR: 3.489 (95\% CI: 1.447-8.413)] were independent prognostic factors. According to the survival curve of independent prognostic factors, the 3 -year survival of patients with high differentiation and high miR-223-3p expression was significantly higher than that of patients with poor differentiation and low miR-223-3p expression $(\mathrm{P}<0.05)$ (Table II and Fig. 2).

\section{Discussion}

Preoperative induction chemotherapy has been proposed in recent years as a treatment program. The adoption of TPF program before the operation can control cancer cell proliferation and interfere with cell biology functions, thereby reducing the volume of solid tumors and facilitating surgical operations (22). Presently, indexes of short-term clinical efficacy evaluation after TPF treatment are scarce. The monitoring of related indexes to predict the short-term clinical efficacy and survival will improve the prognosis of patients.

A growing number of studies have established the close relationship between the miR and the occurrence and development of various tumors (23). miR-223-3p has low expression in a variety of tumors $(24,25)$. In the study by Ding et al $(26)$, miR-223-3p was reported to have low expression in glioblastoma and the overexpression of miR-223-3p can effectively reduce inflammation-associated cytokines in glioblastoma to inhibit cell proliferation and migration. However, little is known about the relationship between miR-223-3p and OSCC. In the present study, we found the serum expression of miR-223-3p in OSCC patients was significantly lower than in healthy people, which is consistent with the findings of Tachibana et al (16). The present study and that of Tachibana et al support each other, but this study is more representative and has a larger sample size. The expression of miR-223-3p after treatment was also monitored in the present study. The results demonstrated that the expression of miR-223-3p after the treatment was significantly higher than before, suggesting that the expression of miR-223-3p can be improved after TPF treatment, but its specific mechanism is not clear. The correlation analysis revealed that the expression of miR-223-3p before the treatment gradually increased with the improvement of treatment outcome, indicating that miR-223-3p expression before the treatment can function as an indicator of the efficacy of OSCC patients. We divided patients into the remission and non-remission group and found that the expression of miR-223-3p before treatment was significantly higher in the remission group than in the non-remission group. The ROC curve analysis suggested that the area under the curve of the miR-223-3p expression before the treatment for OSCC efficacy prediction was 0.797 , with high predictive value. Previous literature $(27,28)$ states that the high expression of miR-223-3p can increase the sensitivity of cancer cells to cisplatin, docetaxel, and 5-FU. This may be one of the reasons why the efficacy in the remission group was better than in the non-remission group in this study, but the specific mechanism needs to be further studied.
Cox regression was conducted to analyze the factors affecting the prognosis of patients. The analysis showed that miR-223-3p and degree of differentiation were independent factors influencing the prognosis of patients. One previous study discovered that the degree of differentiation is related to the prognosis of patients with OSCC (29). However, previous studies mostly focused on the difference between poor differentiation and moderate + high differentiation. The present study made a comparison between moderate differentiation and high differentiation and believed that, like high differentiation, moderate differentiation also has an impact on the prognosis of patients. However, in the present study, the prognosis of oral cancer patients was found to be related with pathological staging but not related with the TNM staging, which is consistent with the results in the study by Chen et al (30). We speculate that this may be the consequence of a small sample size. This study is the first to discover that miR-223-3p can be used as a potential independent prognostic indicator for OSCC.

The present study and above-mentioned studies have proven the clinical value of miR-223-3p in patients with OSCC. However, the specific mechanism of miR-223-3p were not explored and the small sample size in this study requires further study for confirmation.

In summary, miR-223-3p, expression is low in oral cancer, and it shows potential for predicting the efficacy and prognosis of patients with oral squamous cell carcinoma (OSCC) after TPF regimen.

\section{Acknowledgements}

Not applicable.

\section{Funding}

No funding was received.

\section{Availability of data and materials}

The datasets used and/or analyzed during the present study are available from the corresponding author on reasonable request.

\section{Authors' contributions}

$\mathrm{CL}$ and YF conceived and designed the study, and drafted the manuscript. CL, YF and WS collected, analyzed and interpreted the experimental data. CL revised the manuscript for important intellectual content. All authors read and approved the final manuscript.

\section{Ethics approval and consent to participate}

The study was approved by the Ethics Committee of the Affiliated Stomatological Hospital of Jiamusi University (Jiamusi, China). Signed informed consents were obtained from the patients and/or the guardians.

\section{Patient consent for publication}

Not applicable. 


\section{Competing interests}

The authors declare that they have no competing interests.

\section{References}

1. Chi AC, Day TA and Neville BW: Oral cavity and oropharyngeal squamous cell carcinoma - an update. CA Cancer J Clin 65 401-421, 2015.

2. Li L, Li C, Wang S, Wang Z, Jiang J, Wang W, Li X, Chen J, Liu K, Li C, et al: Exosomes derived from hypoxic oral squamous cell carcinoma cells deliver miR-21 to normoxic cells to elicit a prometastatic phenotype. Cancer Res 76: 1770-1780, 2016.

3. Torre LA, Bray F, Siegel RL, Ferlay J, Lortet-Tieulent J and Jemal A: Global cancer statistics, 2012. CA Cancer J Clin 65: 87-108, 2015

4. Tseng WT, Chiang WF, Liu SY, Roan J and Lin CN: The application of data mining techniques to oral cancer prognosis. J Med Syst 39: 59, 2015.

5. Adami GR, Tang JL and Markiewicz MR: Improving accuracy of RNA-based diagnosis and prognosis of oral cancer by using noninvasive methods. Oral Oncol 69: 62-67, 2017.

6. Sharma A, Agarwal K, Agarwal S, Kumar S and Bharti AC: 354P-Study of immunohistochemical expression of VEGF and its association with HPV E6 and E7 oncoproteins in oral and oropharyngeal squamous cell carcinoma. Ann Oncol 28: 100-110, 2017.

7. Smits RW, Koljenović S, Hardillo JA, Ten Hove I, Meeuwis CA, Sewnaik A, Dronkers EA, Bakker Schut TC, Langeveld TP, Molenaar J, et al: Resection margins in oral cancer surgery: Room for improvement. Head Neck 38: 2197-2203, 2016.

8. Zhong LP, Ma H, Ju W and Zhang ZY: Relationship of low stathmin expression and benefit from TPF induction chemotherapy and its role in chemoresistance via mutant p53 in oral cancer. J Clin Oncol 36: e18034, 2018.

9. Zhong LP, Zhang CP, Ren GX, Guo W, William WN Jr, Hong CS, Sun J, Zhu HG, Tu WY, Li J, et al: Long-term results of a randomized phase III trial of TPF induction chemotherapy followed by surgery and radiation in locally advanced oral squamous cell carcinoma. Oncotarget 6: 18707-18714, 2015.

10. Shi J, Bao X, Liu Z, Zhang Z, Chen W and Xu Q: Serum miR-626 and miR-5100 are promising prognosis predictors for oral squamous cell carcinoma. Theranostics 9: 920-931, 2019.

11. Lu J, Getz G, Miska EA, Alvarez-Saavedra E, Lamb J, Peck D, Sweet-Cordero A, Ebert BL, Mak RH, Ferrando AA, et al: MicroRNA expression profiles classify human cancers. Nature 435: 834-838, 2005.

12. Kasinski AL, Kelnar K, Stahlhut C, Orellana E, Zhao J, Shimer E, Dysart S, Chen X, Bader AG and Slack FJ: A combinatorial microRNA therapeutics approach to suppressing non-small cell lung cancer. Oncogene 34: 3547-3555, 2015.

13. Callegari E, Gramantieri L, Domenicali M, D'Abundo L, Sabbioni S and Negrini M: MicroRNAs in liver cancer: A model for investigating pathogenesis and novel therapeutic approaches. Cell Death Differ 22: 46-57, 2015.

14. Hannafon BN, Trigoso YD, Calloway CL, Zhao YD, Lum DH, Welm AL, Zhao ZJ, Blick KE, Dooley WC and Ding WQ: Plasma exosome microRNAs are indicative of breast cancer. Breast Cancer Res 18: 90, 2016.

15. Zeljic K, Jovanovic I, Jovanovic J, Magic Z, Stankovic A and Supic G: MicroRNA meta-signature of oral cancer: Evidence from a meta-analysis. Ups J Med Sci 123: 43-49, 2018.
16. Tachibana H, Sho R, Takeda Y,Zhang X, Yoshida Y,Narimatsu H, Otani K, Ishikawa S, Fukao A, Asao H, et al: Circulating miR-223 in oral cancer: Its potential as a novel diagnostic biomarker and therapeutic target. PLoS One 11: e0159693, 2016.

17. Soga D, Yoshiba S, Shiogama S, Miyazaki H, Kondo S and Shintani S: microRNA expression profiles in oral squamous cell carcinoma. Oncol Rep 30: 579-583, 2013.

18. Kukreja P, Parekh D and Roy P: Practical challenges in measurement of depth of invasion in oral squamous cell carcinoma: Pictographical documentation to improve consistency of reporting per the AJCC 8th edition recommendations. Head Neck Pathol 2019, doi: 10.1007/s12105-019-01047-9.

19. Livak KJ and Schmittgen TD: Analysis of relative gene expression data using real-time quantitative PCR and the $2^{-\Delta \Delta C q}$ method. Methods 25: 402-408, 2001.

20. Necchi A, Lo Vullo S, Perrone F, Raggi D, Giannatempo P, Calareso G, Nicolai N, Piva L, Biasoni D, Catanzaro M, et al: First-line therapy with dacomitinib, an orally available pan-HER tyrosine kinase inhibitor, for locally advanced or metastatic penile squamous cell carcinoma: Results of an open-label, singlearm, single-centre, phase 2 study. BJU Int 121: 348-356, 2018.

21. Hosmer DW Jr, Lemeshow S and Sturdivant RX (eds). Applied Logistic Regression. 3rd edition. John Wiley \& Sons, Hoboken, NJ, pp173-182, 2013

22. Yu CC, Hu FW, Yu CH and Chou MY: Targeting CD133 in the enhancement of chemosensitivity in oral squamous cell carcinoma-derived side population cancer stem cells. Head Neck 38: E231-E238, 2016.

23. Acunzo M, Romano G, Wernicke D and Croce CM: MicroRNA and cancer - a brief overview. Adv Biol Regul 57: 1-9, 2015.

24. Bhattacharya S, Steele R, Shrivastava S, Chakraborty S, Di Bisceglie AM and Ray RB: Serum miR-30e and miR-223 as novel noninvasive biomarkers for hepatocellular carcinoma. Am J Pathol 186: 242-247, 2016

25. Lu W, Hu Y, Ma Q, Zhou L, Jiang L, Li Z, Zhao S, Xu Y, Shi W, Li S, et al: miR-223 increases gallbladder cancer cell sensitivity to docetaxel by downregulating STMN1. Oncotarget 7: 62364-62376, 2016.

26. Ding Q, Shen L, Nie X, Lu B, Pan X, Su Z, Yan A, Yan R, Zhou Y, Li L, et al: MiR-223-3p overexpression inhibits cell proliferation and migration by regulating inflammation-associated cytokines in glioblastomas. Pathol Res Pract 214: 1330-1339, 2018.

27. Bozec A, Zangari J, Butori-Pepino M, Ilie M, Lalvee S, Juhel T, Butori C, Brest P, Hofman P and Vouret-Craviari V: MiR-223-3p inhibits angiogenesis and promotes resistance to cetuximab in head and neck squamous cell carcinoma. Oncotarget 8: 57174-57186, 2017.

28. Zhou X, Jin W, Jia H, Yan J and Zhang G: MiR-223 promotes the cisplatin resistance of human gastric cancer cells via regulating cell cycle by targeting FBXW7. J Exp Clin Cancer Res 34: 28, 2015.

29. Ramos-García P, Bravo M, González-Ruiz L and GonzálezMoles MÁ: Significance of cytoplasmic cyclin D1 expression in oral oncogenesis. Oral Dis 24: 98-102, 2018.

30. Chen TC, Wu CT, Wang CP, Hsu WL, Yang TL, Lou PJ, Ko JY and Chang YL: Associations among pretreatment tumor necrosis and the expression of HIF-1 $\alpha$ and PD-L1 in advanced oral squamous cell carcinoma and the prognostic impact thereof. Oral Oncol 51: 1004-1010, 2015. 